\title{
INFLUENCE OF SCALE PARAMETERS OF PEARLITE ON THE KINETICS OF ANISOTHERMAL PEARLITE-TO-AUSTENITE TRANSFORMATION IN A EUTECTOID STEEL
}

\author{
F. G. CABALLERO ${ }^{1}$, C. CAPDEVILA ${ }^{1,2}$ and C. GARCÍA DE ANDRÉS ${ }^{1}$ \\ ${ }^{1}$ Department of Physical Metallurgy, Centro Nacional de Investigaciones Metalúrgicas (CENIM), Consejo \\ Superior de Investigaciones Científicas (CSIC), Avda. Gregorio del Amo, 8, 28040 Madrid, Spain \\ ${ }^{2}$ Department of Materials Science and Metallurgy, University of Cambridge, Pembroke Street, Cambridge CB2 \\ 3QZ, UK
}

Keywords: steels, kinetics, phase transformations, modeling

\section{Introduction}

Formation of austenite from pearlite is well established to be a nucleation and growth process (1-6). The structural factors affecting the location of nuclei of austenite and determining the rate of nucleation and growth of austenite are necessarily different from those of the reverse transformation, i.e., the formation of pearlite from austenite (7-9). In the case of formation of austenite from aggregates of ferrite and carbides, the most relevant structural factor to be considered is the degree of dispersion of the carbide. In pearlite, the interlamellar spacing may be taken as a measure of the dispersion of the carbide.

In fully pearlitic steel, austenite nucleates heterogeneously at the junctions between pearlite colonies. This is in spite of the relatively large amount of interlamellar surfaces available within the pearlite colonies, which seem to be much less effective as sites for the nucleation of austenite (2). The rate of growth of the austenite is primarily controlled by the rate of carbon diffusion in the austenite between adjacent pearlitic cementite lamellae, but may also be influenced by boundary diffusion of substitutional alloying elements at low temperature (6).

The Avrami's equation is generally used to model transformations under isothermal conditions $(8,10-12)$. However, in this paper, a model is presented in which this equation has been applied successfully in the modeling of the pearlite-to-austenite transformation during continuous heating in a eutectoid steel with a fully pearlitic initial microstructure. Moreover, the influence of the parameters that characterize the scale of pearlite with a lamellar morphology (the interlamellar spacing and the area per unit volume of the pearlite colony interface) on pearlite-to-austenite transformation kinetics has also been studied and considered in the modeling.

\section{Experimental Procedure}

A eutectoid carbon steel with an actual composition (wt. \%) of 0.76C, $0.24 \mathrm{Si}, 0.91 \mathrm{Mn}$ and $0.013 \mathrm{P}$ was used. The following heat treatments were carried out to yield in this steel fully pearlitic microstructures with different scale parameters. Specimens were austenitized for $5 \mathrm{~min}$ at $1273 \mathrm{~K}$, isothermally transformed at three different temperatures and subsequently cooled rapidly to room temperature. Table 1 lists all the temperatures and holding times used for the isothermal formation of pearlite with different scale parameters in this steel.

Specimens were ground and polished using standardized techniques and finished on $0.25 \mu \mathrm{m}$ diamond paste for metallographic examination. An etching solution of picric acid in isopropyl alcohol with several drops of Vilella's reagent was used to disclose pearlite in specimens S1and S2 on a JEOL JXA-820 scanning electron microscope (SEM). Pearlite in specimen S3 was revealed by transmission electron microscopy (TEM). For this, $3 \mathrm{~mm}$ diameter cylindrical samples were sliced into $100 \mu \mathrm{m}$ thick discs and subsequently ground down to foils of $50 \mu \mathrm{m}$ thickness on wet 800 grit silicon carbide paper. These foils were finally electropolished until 
perforation occurred in a twin-jet electropolisher (E. A. Fischione Inst. Mfg - Model 110) at room temperature and a voltage of $100 \mathrm{~V}$ using a solution of $5 \%$ perchloric acid, $15 \%$ glycerol and $80 \%$ methanol. The foils were examined in a JEOL - 200CX transmission electron microscope at an operating voltage of $200 \mathrm{kV}$.

TABLE 1

\section{$\underline{\text { Results and Discussion }}$}

Figure 1 shows scanning (S1 and S2) and transmission (S3) electron micrographs of the three different scales of pearlite studied in this steel.
(a) S1. SEM
(b) S2. SEM

(c) S3. TEM

Figure 1. Electron micrographs of the three different scales of pearlite considered in this study.

Two parameters, the mean true interlamellar spacing $\left(\sigma_{o}\right)$ and the area per unit volume of the pearlite colonies interface $\left(S_{v}^{P P}\right)$, characterize the scale of pearlite with a lamellar morphology (3). The values of $\sigma_{o}$ were derived from electron micrographs according to Underwood's intersection procedure (13). The values of $S_{v}^{P P}$ were measured as reported by Roosz et al. (3). 
Approximating the pearlite colony by a truncated octahedron, the edge length of the pearlite colonies $\left(a^{P}\right)$ is calculated from the area per unit volume $S_{v}^{P P}$ with the following expression (14):

$$
S_{v}^{P P}=\frac{6(1+2 \sqrt{3})\left(a^{P}\right)^{2}}{8 \sqrt{2}\left(a^{P}\right)^{3}}=\frac{3(1+2 \sqrt{3})}{4 \sqrt{2} a^{P}}
$$

Results for $\sigma_{o}, S_{v}^{P P}$ and $a^{P}$ are listed in Table 2. Since recent recalculations have been performed, results for S2 specimen quoted in authors previous publication (15) slightly deviate for those presented in Table 2.

\author{
TABLE 2
}

The experimental validation of the austenite formation kinetics model developed in this work was carried out using an Adamel Lhomargy DT1000 high-resolution dilatometer. To analyze the progress of pearlite-toaustenite transformation, interrupted heating experiments were carried out by quenching. Dilatometric specimens with three different scales of lamellar pearlite were heated at a constant rate of $0.5 \mathrm{Ks}^{-1}$. Each test was repeated three times. The heating dilatometric curves of the samples were analyzed to determine the start temperature $\left(A c_{1}\right)$ and the end temperature $\left(A c_{3}\right)$ of pearlite-to-austenite transformation and then several quench-out temperatures were selected in order to analyze the progress of pearlite-to-austenite transformation. Table 3 lists the selected temperatures at which heating was interrupted by quenching for each scale of pearlite: $A c_{1}-5 \mathrm{~K}\left(\mathrm{~T}_{\mathrm{a}}\right), \mathrm{Ac}_{1}\left(\mathrm{~T}_{\mathrm{b}}\right)$, the temperatures at the maximum $\left(\mathrm{T}_{\mathrm{c}}\right)$, inflexion point $\left(\mathrm{T}_{\mathrm{d}}\right)$ and minimum $\left(\mathrm{T}_{\mathrm{e}}\right)$ of the heating dilatometric curve, $\mathrm{Ac}_{3}\left(\mathrm{~T}_{\mathrm{f}}\right)$ and $\mathrm{Ac}_{3}+10 \mathrm{~K}\left(\mathrm{~T}_{\mathrm{g}}\right)$. The $\mathrm{Ac}_{1}$ and $\mathrm{Ac}_{3}$ temperatures are the average value of three tests. Austenite, which is formed inside pearlite, transforms to martensite during quenching. Thus, the progress of pearlite-to-austenite transformation is determined throughout the evolution of the volume fraction of martensite.

Specimens were ground and polished using standardized metallographic techniques. Le Pera's reagent (16) was used to reveal martensite formed during quenching. The quantitative measurement of martensite volume fraction was determined by point counting (13).

TABLE 3 
Nucleation and growth processes under isothermal condition can be described in general using the Avrami's equation (17):

$x=1-\exp \left(-K t^{n}\right)$

where $x$ represents the formed austenite volume fraction, $K$ is a constant for a given temperature, $t$ is the time and $n$ is a constant characterizing the kinetics. Roosz et al. (3) obtained a value of $n=4$ from their measured data under isothermal condition. According to Christian (18), with a spherical configuration, a value of $n=4$ means that the nucleation rate $(\dot{N})$ and the growth rate $(G)$ are constant in time. This gives a transformed volume fraction of:

$x=1-\exp \left(-\frac{\pi}{3} \dot{N} G^{3} t^{4}\right)$

Roosz et al. (3) proposed a temperature and structure dependence of $\dot{N}$ and $G$ as a function of the reciprocal value of overheating $\left(\Delta T=T-A c_{1}\right)$ as follows:

$$
\begin{aligned}
& \dot{N}=f_{N} \exp \left(\frac{-Q_{N}}{k \Delta T}\right) \\
& G=f_{G} \exp \left(\frac{-Q_{G}}{k \Delta T}\right)
\end{aligned}
$$

where $Q_{N}$ and $Q_{G}$ are the activation energies of nucleation and growth, respectively, $k$ is Boltzmann's constant, and $f_{N}$ and $f_{G}$ are the functions representing the influence of the structure on the nucleation and growth rates, respectively. The activation energies of nucleation and growth, $Q_{N}$ and $Q_{G}$, were determined experimentally by Roosz et al. (3) in a eutectoid steel $\left(Q_{N}=3.5 \times 10^{-22} \mathrm{~J}\right.$ and $\left.Q_{G}=4.1 \times 10^{-22} \mathrm{~J}\right)$.

The three different ferrite/cementite interface sites for austenite nucleation in fully pearlitic steels are: A) the interface of ferrite and cementite platelets, B) the surfaces of the pearlite colony, and C) the triple points of the pearlite colony intersections. Several authors (2-4) reported that the nucleation of austenite inside pearlite takes place preferentially at the points of intersection of cementite with the edges of the pearlite colony (type C). Approximating the pearlite colony as a truncated octahedron, the number of nucleation sites per unit volume (type C) is calculated as follows:

$N_{C}=\frac{\left(\frac{36}{3} \frac{a^{P}}{\sigma_{o}}\right)}{8 \sqrt{2}\left(a^{P}\right)^{3}}=\frac{36 a^{P}}{24 \sqrt{2}\left(a^{P}\right)^{3} \sigma_{o}} \approx \frac{1}{\left(a^{P}\right)^{2} \sigma_{0}}$

where $a^{P}$ is the edge length of the pearlite colony and $\sigma_{o}$ is the interlamellar spacing.

Bearing in mind that the rate of nucleation increases as the pearlite interlamellar spacing decreases and the edge length of the pearlite colony increases (1), the function $f_{N}$ in equation [4] would have the general form:

$$
f_{N}=K_{N} \frac{\left(a^{P}\right)^{n}}{\sigma_{o}^{m}}\left(N_{C}\right)^{i}
$$

where $K_{N}, n, m$, and $i$ are empirical parameters. These parameters were adjusted in order to obtain good fit between theory and the experimental austenite volume fraction curves. Different cases of structure dependence 
for $n, m$, and $i$ were investigated and it was found out that the measured values of austenite volume fraction as a function of temperature can be best described with $n=6, m=1$, and $i=\frac{1}{6}$ (19).

Moreover, if the growth of austenite is considered to be controlled by interface diffusion of substitutional elements (3), the function $f_{G}$ in equation [5] representing the structure dependence on the growth rate can be expressed as follows:

$$
f_{G}=K_{G} \frac{1}{\sigma_{0}^{2}}
$$

where $K_{G}$ is an empirical constant.

Substituting equations [7] and [8] in equations [4] and [5], respectively:

$$
\begin{aligned}
& \dot{N}=7 \times 10^{11} \frac{\left(a^{P}\right)^{6}}{\sigma_{o}}\left(N_{C}\right)^{\frac{1}{6}} \exp \left(\frac{-25.38}{\Delta T}\right) \quad \text { in }\left(1 / \mathrm{mm}^{3} \mathrm{~s}\right) \\
& G=1 \times 10^{-10} \frac{1}{\sigma_{0}^{2}} \exp \left(\frac{-29.7}{\Delta T}\right) \quad \text { in }(\mathrm{mm} / \mathrm{s})
\end{aligned}
$$

Figure 2. Experimental and calculated results for the formation of austenite inside pearlite under continuous heating conditions in a eutectoid steel.

The difficulties in treating non-isothermal reactions are meanly due to the independent variations of growth and nucleation rates with temperature, described in equations [9] and [10]. The problem is only undertaken when the rate of transformation depends exclusively on the state of the assembly and not on the thermal path by which the state is reached (18). Reactions of this type are called isokinetic. Avrami defined an isokinetic reaction by the condition that the nucleation and growth rates are proportional to each other (i.e. they have the same temperature variation). This leads to the concept of additivity and Scheil's rule (20).

Since Avrami's condition for an isokinetic reaction is not here satisfied, a general equation to describe the nonisothermal overall pearlite-to-austenite transformation in pearlitic steel was derived elsewhere (15) integrating the Avrami's equation over the whole temperature range where the transformation takes place. 
$x=1-\exp \left(-\int_{A c_{1}}^{T} \frac{4 \pi}{3(\dot{T})^{4}} \dot{N} G^{3} \Delta T^{3} d T\right)$

where $\dot{T}$ is the heating rate and, $\dot{N}$ and $G$ are given by equations [9] and [10], respectively. The eutectoid temperature $A c_{1}$ of the steel was calculated using Andrews' formula (21).

Figure 2 shows the experimental and calculated austenite formation kinetics plotted as a function of temperature. From this diagram it can be seen that the eutectoid reaction (pearlite $\rightarrow$ austenite) proceeds within a narrow temperature range. The figure suggests that austenite transformation starts at higher temperature the coarser the pearlite. On the other hand, the kinetics of the transformation is slower the coarser the initial pearlite microstructure.

Likewise, from Fig. 2 can be concluded that calculated austenite volume fraction was consistent with the measured value for all different scales of pearlite.

\section{Conclusions}

Since conditions to apply Scheil's rule are rarely satisfied, a mathematical model applying the Avrami's equation has been used to reproduce the kinetics of the pearlite-to-austenite transformation in a eutectoid steel during continuous heating. The model proposes two functions, $f_{N}$ and $f_{G}$, which represent the dependence of nucleation and growth rates, respectively, on the structure. The influence of the parameters that characterize the scale of pearlite with a lamellar morphology on pearlite-to-austenite transformation kinetics has been also experimentally studied. It has been found that pearlite-to-austenite transformation commences at higher temperature the coarser the pearlite. Moreover, the kinetics of the transformation is slower the coarser the initial pearlite microstructure. Experimental results for the austenite volume fraction are in good agreement with the predicted values from the model proposed in this work.

\section{$\underline{\text { Acknowledgements }}$}

The authors acknowledge financial support from Consejería de Educación y Cultura de la Comunidad Autónoma de Madrid (CAM 07N/0065/1998).

\section{References}

1. Roberts, GA and Mehl, RF, Trans. ASM. 1943; 31: 613.

2. Speich, GR and Szirmae, A, Trans. TMS-AIME. 1969; 245: 1063.

3. Roosz, A, Gacsi, Z and Fuchs, EG, Acta Metall. 1983; 31: 509.

4. Judd, RR and Paxton, HW, Trans. TMS-AIME, 1968; 242: 206.

5. Hillert, M, Nilsson, K and Torndahl, LE, Journ. of the Iron and Steel Inst. 1971; 209: 49.

6. Speich, GR, Demarest, VA and Miller, RL, Metall. Trans. 1981; 12A: 1419.

7. Zener, C, Trans. AIME. 1946; 167: 550.

8. Hillert, M, Jernkont. Ann. 1957; 141: 757.

9. Hillert, M, The Mechanism of Phase Transformation in Crystalline Solids. London: Institute of Metals, 1969: 231.

10. Denis, S, Farias, D and Simon, A, ISIJ International. 1992; 32: 316.

11. Reed, RC and Bhadeshia, HKDH, Mater. Sci. Technol. 1992; 8: 421.

12. Grong, O, Metallurgical Modelling of Welding. Eds. Bhadeshia, HKDH. London: The Institute of Metals, 1995: 187.

13. Underwood, EE, Quantitative Stereology. Reading: Addison-Wesley Publishing Co., 1970: 73.

14. De Hoff, RT and Rhines, FH, Quantitative Stereology. New York: McGraw-Hill, 1968: 93.

15. García de Andrés, C, Caballero, FG, Capdevila, C and Bhadeshia, HKDH, Scripta Materialia. 1998; 39: 791.

16. Le Pera, FS, Journ. of Metals. 1980; 32: 38.

17. Avrami, M, J. Chem. Phys. 1940; 8: 212. 
18. Christian, JW, The Theory of Transformations in Metals and Alloys. Oxford: Pergamon Press, 1975: 19.

19. Caballero, FG, Modelización de las Transformaciones de Fase en Calentamiento Continuo de Aceros con Microestructura Inicial de Ferrita, Perlita y Ferrita-Perlita. PhD Thesis. Madrid: Universidad Complutense de Madrid, 1999: 56.

20. Kamat, RG, Hawbolt, EB, Brown, LC and Brimacombe, JK, Metall. Trans. 1992; 23A: 2469.

21. Andrews, KW, JISI. 1965; 203: 721. 
TABLE 1. Isothermal Conditions for the Formation of Fully Pearlitic Microstructures

\begin{tabular}{lcc}
\hline Specimen & Temperature (K) & Time (min) \\
\hline S1 & 948 & 45 \\
S2 & 923 & 10 \\
S3 & 798 & 60 \\
\hline
\end{tabular}

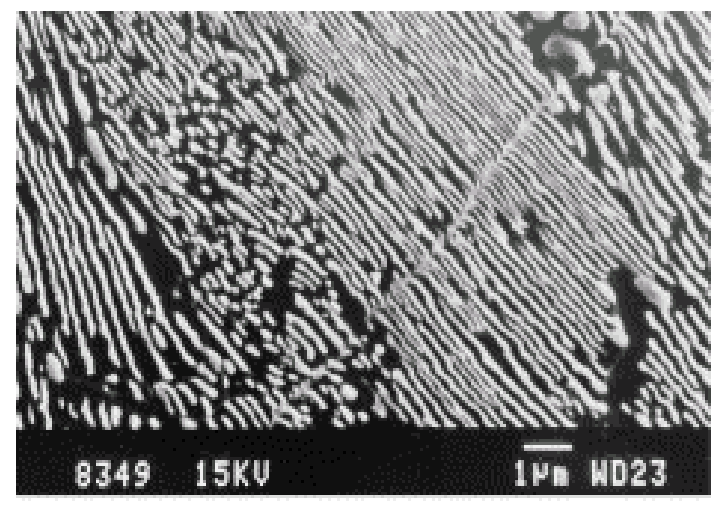

(a)

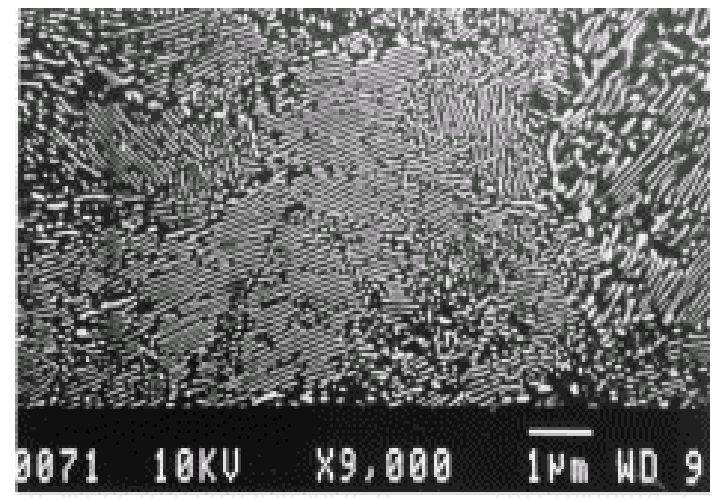

(b)

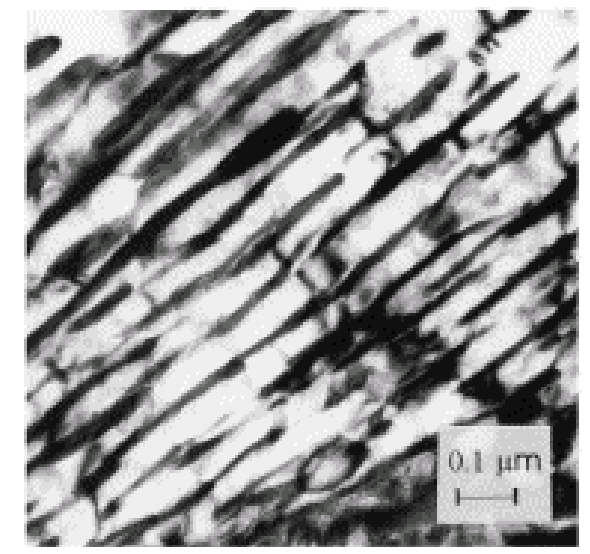

(c)

Figure 1. Electron micrographs of the three different scales of pearlite considered in this study. 
TABLE 2. Scale Characterization of Pearlite

\begin{tabular}{lccr}
\hline Specimen & $\sigma_{o} \times 10^{-3}(\mathrm{~mm})$ & $S_{v}^{P P}\left(\mathrm{~mm}^{-1}\right)$ & $a^{P} \times 10^{-3}(\mathrm{~mm})$ \\
\hline S1 & $0.20 \pm 0.03$ & $581 \pm 86$ & $4.16 \pm 0.70$ \\
S2 & $0.08 \pm 0.01$ & $1399 \pm 273$ & $1.76 \pm 0.34$ \\
S3 & $0.06 \pm 0.01$ & $1432 \pm 60$ & $1.65 \pm 0.07$ \\
\hline
\end{tabular}

TABLE 3. Temperatures in K of Heating Interruption by Quenching

\begin{tabular}{lclccccc}
\hline Specimen & $\mathrm{T}_{\mathrm{a}}=\mathrm{Ac}_{1}-5 \mathrm{~K}$ & $\mathrm{~T}_{\mathrm{b}}=\mathrm{Ac}_{1}$ & $\mathrm{~T}_{\mathrm{c}}$ & $\mathrm{T}_{\mathrm{d}}$ & $\mathrm{T}_{\mathrm{c}}$ & $\mathrm{T}_{\mathrm{f}}=\mathrm{Ac}_{3}$ & $\mathrm{~T}_{\mathrm{g}}=\mathrm{Ac}_{3}+10 \mathrm{~K}$ \\
\hline $\mathrm{S} 1$ & 1005 & 1010 & 1011 & 1018 & 1026 & 1031 & 1041 \\
S2 & 999 & 1004 & 1006 & 1012 & 1019 & 1020 & 1030 \\
S3 & 998 & 1003 & 1006 & 1011 & 1017 & 1020 & 1030 \\
\hline
\end{tabular}




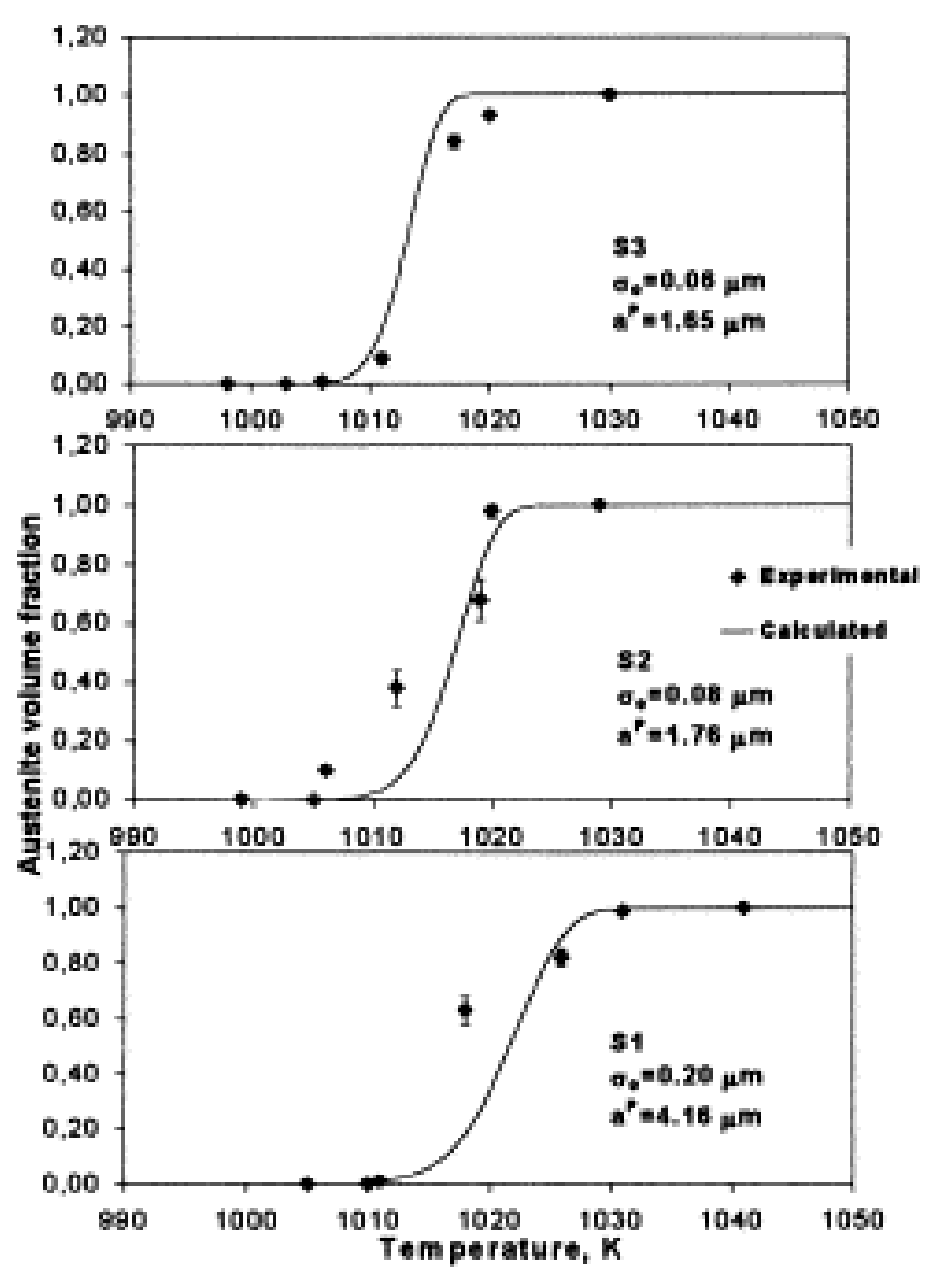

Figure 2. Experimental and calculated results for the formation of austenite inside pearlite under continuous heating conditions in a eutectoid stee 